13. Vidal-Madjar, A., Ferlet, R. \& Lemoine, M. Deuterium abundance and cosmology. Preprint astro-ph/ 9612020

14. Pagel, B. E. J. Nucleosynthesis and Chemical Evolution of Galaxies (Cambridge Univ. Press, 1997).

15. Schramm, D. S. \& Turner, M. S. Deuteronomy and numbers. Nature 381, 193-194 (1996).

16. Hata, N., Steigman, G., Bludman, S. \& Langacker, P. Cosmological implications of two conflicting deuterium abundances. Phys. Rev. D 55, 540-547 (1997).

17. Vangioni-Flam, E. \& Cassé, M. Cosmological and astrophysical consequences of a high primordial deuterium abundance. Astrophys. J. 441, 471-476 (1995).

18. Hogan, C. J. Big bang nucleosynthesis and the observed abundances of light elements. Preprint astro$\mathrm{ph} / 9609138$.

19. Copi, C. J., Olive, K. A. \& Schramm, D. N. Implications of a primordial origin for the dispersion in D/ $\mathrm{H}$ in quasar absorption systems. Preprint astro-ph/9606156.

20. Copi, C. J., Schramm, D. N. \& Turner, M. S. Big-bang nucleosynthesis and the baryon density of the universe. Science 267, 192-199 (1995).

21. Jedamzik, K. \& Fuller, G. M. Nucleosynthesis in the presence of primordial isocurvature baryon fluctuations. Astrophys. J. 452, 33-61 (1995).

22. Jedamzik, K. \& Fuller, G. M. Is the deuterium in high redshift Lyman limit systems primordial? Preprint astro-ph/9609103.

23. White, M., Viana, P. T. P., Liddle, A. R. \& Scott, D. Cold dark matter models with high baryon content. Mon. Not. R. Astron. Soc. 283, 107-118 (1996).

24. White, S. D. M., Navaro, J. F., Evrard, A. E. \& Frenk, C. S. The baryon content of galaxy clusters: a challenge to cosmological orthodoxy. Nature 366, 429-433 (1993).

Acknowledgements. This work is based on observations with the NASA/ESA Hubble Space Telescope, obtained at the Space Telescope Science Institute, which is operated by the Association of Universities fo Research in Astronomy, Inc., under NASA contract. K.M.L. was supported by NASA, STScI and NSF; M.L. was supported by NASA, DoE and NSF at the University of Chicago; J.K.W. thanks M. Ashley, J. Barrow, A. Little and R. J. Tayler for discussions, and SUN Microsystems Australia Pty Ltd for providing computing facilities for this work.

\section{Chaotic dynamics of falling disks}

\author{
Stuart B. Field ${ }^{\star}$, M. Klaus $\dagger$, M. G. Moore $\dagger \ddagger$ \\ \& Franco Nori $\dagger$
}

* Department of Physics, Colorado State University, Fort Collins, Colorado 80523, USA

$\dagger$ Department of Physics, The University of Michigan, Ann Arbor, Michigan 48109-1120, USA

The study of the motion of flat bodies falling in a viscous medium dates back at least to Newton ${ }^{1}$ and Maxwell ${ }^{2}$, and is relevant to problems in meteorology $y^{3}$, sedimentology ${ }^{4}$, aerospace engineering $^{1}$ and chemical engineering ${ }^{5-8}$. More recent theoretical studies $^{9-12}$ have emphasized the role played by deterministic chaos, although many experimental studies ${ }^{1,5-8,13,14}$ were performed before the development of such ideas. Here we report experimental observations of the dynamics of disks falling in water/glycerol mixtures. We find four distinct types of motion, which are mapped out in a 'phase diagram'. The apparently complex behaviour can be reduced to a series of one-dimensional maps, which display a discontinuity at the crossover from periodic to chaotic motion. This discontinuity leads to an unusual intermittency transition $^{15}$, not previously observed experimentally, between the two behaviours.

Steel and lead disks with diameters $d=5.1-18.0 \mathrm{~mm}$ and thicknesses $t=0.076-1.63 \mathrm{~mm}$ were dropped in water and water/glycerol mixtures from heights ranging from $\sim 0.3$ to $1 \mathrm{~m}$; some paper disks were also dropped in air, as discussed later. This combination of disk sizes and densities, and fluid viscosities, allowed us to explore a very large region of parameter space. Several different types of motion were observed (Fig. 1).

We now consider the system parameters that determine the type of motion observed. There are five material parameters: the disk diameter $d$, thickness $t$ and density $\rho$, as well as the fluid density $\rho_{\mathrm{f}}$ and kinematic viscosity $\nu$. From these five, three independent dimensionless ratios ${ }^{13}$ may be formed. The first is the dimensionless moment of inertia $I^{*}=I_{\text {disk }} / \rho_{\mathrm{f}} d^{5}=\pi \rho t / 64 \rho_{\mathrm{f}} d$. For small $I^{*}$, we

$\ddagger$ Present address: Center for Nonlinear Dynamics and Department of Physics, University of Texas at Austin, Austin, Texas 78712, USA. expect the effects of the fluid to be important; at large $I^{*}$ the moment of inertia of the disk dominates. A second dimensionless quality is the Reynolds number $\operatorname{Re}=U d / \nu$, where $U$ is the mean vertical disk velocity. Finally, for the disks we consider here, the quantity $t / d$ is small, and we expect therefore that this ratio plays no role in the discs' dynamics.

To investigate the dependence of the disks' behaviour on two dimensionless parameters $I^{*}$ and Re, we have dropped a large number of disks and visually characterized their motion as 'steady falling', 'periodic oscillating', 'chaotic' or 'tumbling'. We may then plot a 'phase' diagram, indicating the behaviour of a disk as a function of $I^{*}$ and $\operatorname{Re}$ (Fig. 2). Also plotted on this diagram are data from refs 13 and 14, which agree quite well with ours. We note that there is a well defined boundary between each regime. This implies that the two dimensionless quantities $I^{*}$ and $\operatorname{Re}$ do in fact characterize a disk/liquid combination.

Mapping out a phase diagram by direct observation allows a compact overview of the disks' behaviour. However, it does not give us any physical insight as to why the disks fall as they do. To explore this question more fully, we have performed direct video imaging of the trajectories of disks as they fall. From such images, it is possible to obtain the two cartesian coordinates of a disk's centre in the vertical plane perpendicular to the direction of observation, as well as the direction in space of the symmetry axis of the disk. The cartesian coordinate parallel to the direction of observation, and the angle of rotation around the symmetry axis, could not be recorded. A simple analysis of this motion would seem difficult, then, as the disk has six degrees of freedom, of which we record four.

We have found it possible to describe this complex behaviour by a simple one-dimensional map. The single variable required is the angle $\theta$ between the disk's normal and the vertical. To understand this, imagine dropping a disk from rest in a fluid, starting at an angle $\theta_{0}$. As seen in Fig. 1a, the disk will glide downwards and to the side, level off, and then begin to tilt in the other direction. Just when it has reached the extremum of its motion (indicated by the arrow in Fig. 1a) the disk is at a well-defined final angle $\theta_{\mathrm{f}}$. As the motion is deterministic, this final angle $\theta_{\mathrm{f}}$ must be a function $f$ of its initial conditions - the initial angle equalling $\theta_{0}$ and the other coordinates and velocities being zero. Thus, it must be true that $\theta_{\mathrm{f}}=f\left(\theta_{0}\right)$.

We may now ask the question as to whether this final angle $\theta_{\mathrm{f}}$ may serve as the initial angle $\theta_{\mathrm{i}}$ for the next oscillation, with the next $\theta_{\mathrm{f}}$ determined from the same mapping $f$ as above. This will be possible to the extent to which this angle alone completely specifies the initial conditions, even for a disk which has fallen quite far. How might this be so? At an extremum (Fig. 1) the angular velocities are essentially zero. So, too, are the horizontal translation velocities. The vertical velocity, although not zero, very rapidly reaches a terminal value which is about the same for all subsequent extrema. Certainly the disk's centre-of-mass coordinates are irrelevant if we consider the fluid to be infinite. We also expect from symmetry considerations that effects of rotations around the disk's axis will be small. Thus, the only relevant disk coordinate is the angle $\theta$ between the disk's plane and the horizontal.

The effect of the inifinite degrees of freedom of the fluid is, of course, much more difficult to consider. A detailed study of the fluid dynamics as the disk approaches a given $\theta_{\mathrm{f}}$ would undoubtedly reveal vortex shedding, turbulence and other complex motion. Without knowing the details of the motion of the fluid, however, we can imagine that for a repetitive motion like the periodic oscillation of a disk, the motion of the fluid in the vicinity of the disk also repeats itself. Thus, we can consider the fluid motion to be included in our description of the state of the system, which we describe by the single variable $\theta_{\mathrm{f}}$. This state is still determined by the previous $\theta_{\mathrm{i}}$, even though it is determined by extremely complicated dynamics. We can thus think of $f\left(\theta_{\mathrm{i}}\right)$ as a single-valued function, and can map it out empirically.

This idea of including the fluid dynamics in some variable which 
ostensibly describes the disk is not a novel one. For the related case of a body moving in an ideal fluid, Kirchhoff showed (see, for example, ref. 9) that the equations of motion for such a body can be written so that the infinite degrees of freedom of the fluid are eliminated; they still influence the body dynamics by way of the added mass tensor, whose elements depend only on the shape of the body.
In Fig. 3, we show eight experimental iteration maps of $\theta_{\mathrm{f}}$ versus $\theta_{\mathrm{i}}$, for several disk/liquid combinations. Maps shown in Fig. 3a-d correspond to those points in the phase diagram of Fig. 2 labelled ad. We see that the latter form a vertical slice in phase space which cuts from the periodic-oscillation region of low $I^{*}$ through the boundary with the chaotic region of higher $I^{*}$. The progression of the

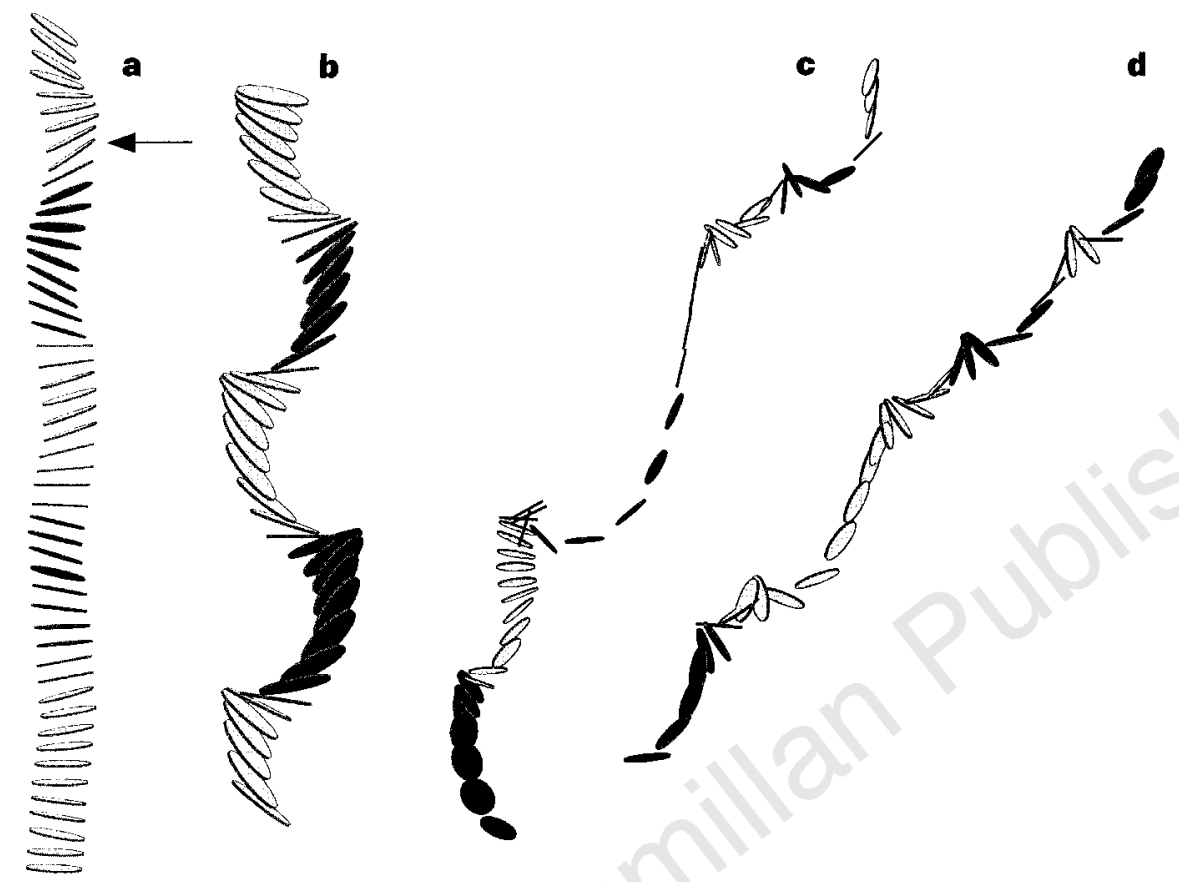

Figure 1 Trajectories of falling disks, obtained by imaging from the side using a video camera; the digitized images were measured, and computerdrawn images are shown. One side of each disk is drawn white, the other black. a, Steady-falling regime observed at lower Reynolds' numbers (Re): a disk, dropped with any initial orientation, quickly settles down to a steady fall with horizontal orientation. The arrow represents an extremum of motion. b. Periodic-oscillating motion observed at higher Re and low dimensionless moment of inertia $l^{*}$. Such disks oscillate with a well-defined period, again settling into this pattern after initial transients have died down. c, Chaotic motion found when both Re and $l^{*}$ are moderately large. Typically, a disk in this regime will begin to oscillate with larger and larger amplitude until its angle is so high that it actually flips over. It then tumbles several times and then suddenly jumps back to oscillating behaviour. The number of tumbles and time between tumbling behaviours appear to the eye to be random. The disk may also suddenly change its overall direction of motion. $\mathbf{d}$, Tumbling motion found at very large $/^{*}$. Here, the disk turns continuously end-over-end while drifting in one direction.

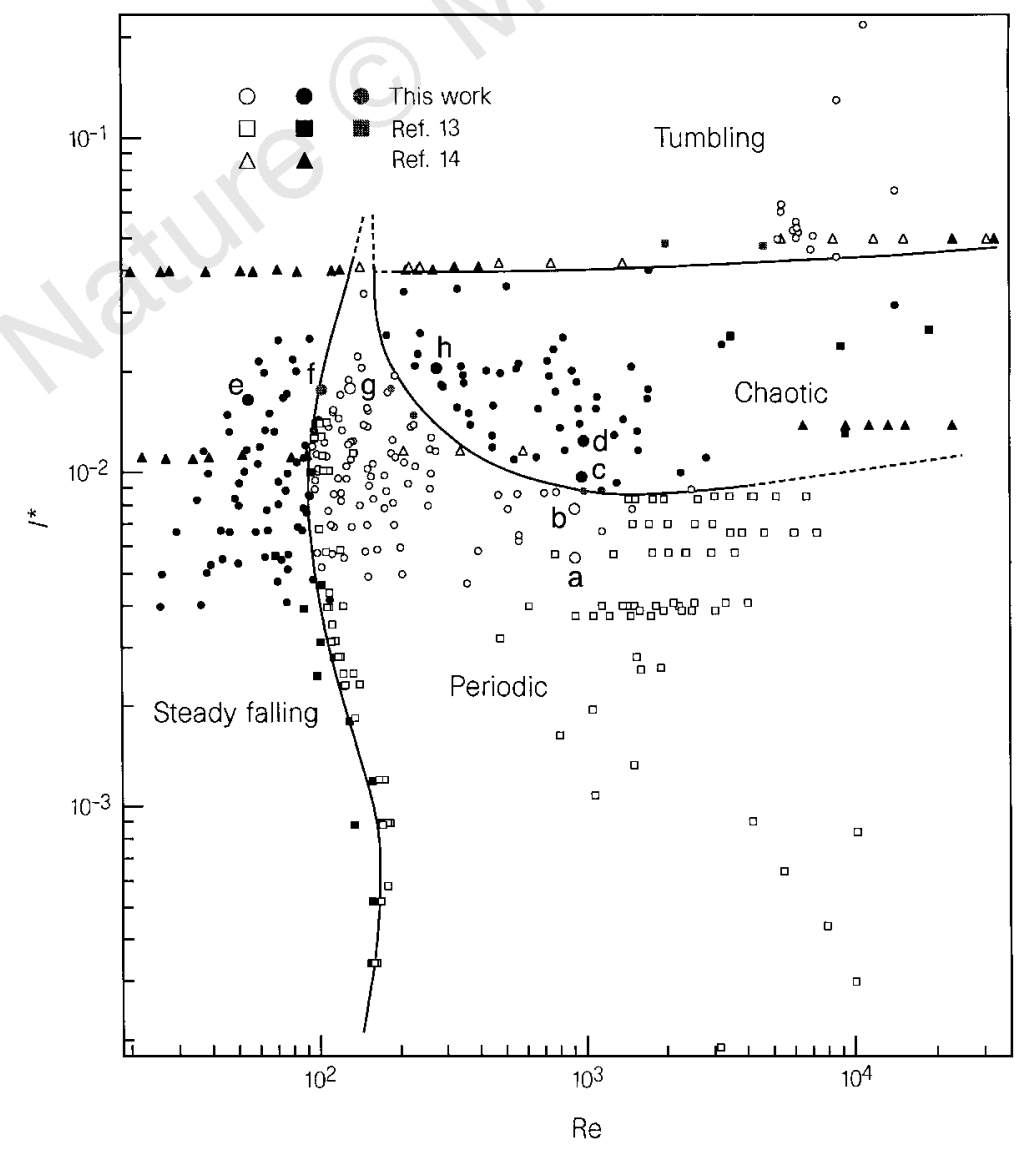

Figure 2 Phase diagram showing the dynamical behaviour of the disks as a function of the two parameters $/^{*}$ (dimensionless moment of inertia) and Re (Reynold's number). Filled symbols represent disks with steady falling or chaotic behaviour, and open symbols periodic or tumbling behaviour. Grey symbols represent cases judged to lie on the border between two regimes. The lettered points, enlarged for clarity, correspond to iteration maps shown in Fig. 3a-h. 
maps indicates how this change in dynamical behaviour proceeds. In Fig. 3a we see that, near an angle of about $\theta_{0} \approx 60^{\circ}$, the map cuts across the diagonal line $\theta_{\mathrm{f}}=\theta_{\mathrm{i}}$ with a slope less than one. $\theta_{0}$ thus represents a stable fixed point, and the motion will always settle down to periodic oscillations with an extremum angle of about $60^{\circ}$. As we increase $I^{*}$, we come to point b in Fig. 2, which is near the border between periodic and chaotic behaviour. In the iteration map of Fig. 3b, we see that there appears to be a stable fixed point, although it is very near to $90^{\circ}$. Thus, at the end of each oscillation, the disk is nearly vertical, and is quite near to actually flipping over. Increasing $I^{*}$ further, we come to point $\mathrm{c}$ in Fig. 2, which is now clearly in the chaotic region. The map (Fig. 3c) no longer crosses the diagonal, so there are no fixed points. Instead, a disk dropped with any initial angle very rapidly increases its successive angles until $\theta \geqslant 90^{\circ}$. We see from the map, however, that there is no final angle defined for initial angles greater than about $80^{\circ}$; for initial angles greater than this the disk will actually flip over. We find that it then tumbles several times; this number is unpredictable. The disk is then suddenly reinjected into the map at some low angle $\theta$, and the process begins again. This progression from oscillations near a fixed point to chaotic behaviour has been termed intermittency ${ }^{15}$. Finally, for larger $I^{*}$ (Fig. 3d), the map is very far from the diagonal line, and the motion is strongly chaotic. Oscillation angles rapidly build towards $90^{\circ}$, and the disk alternates rapidly between tumbling and oscillating. We have schematically indicated this complicated tumbling behaviour by the rapidly fluctuating curve for $\theta \geqslant 67^{\circ}$ in Fig.

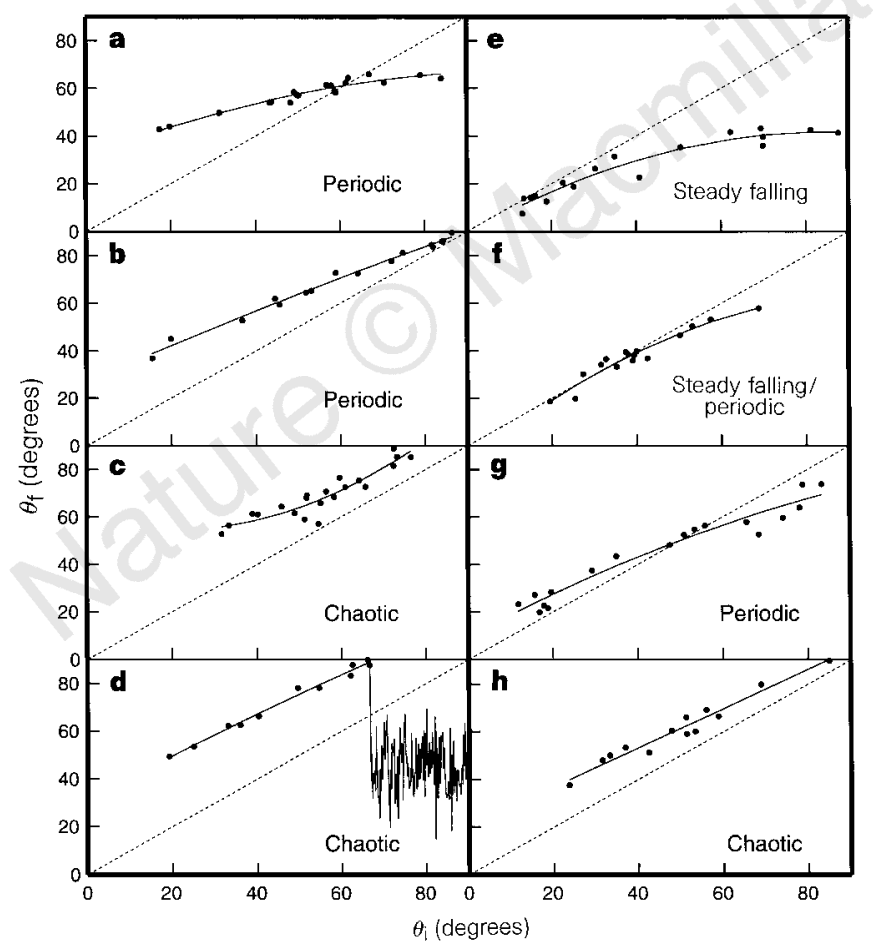

Figure $\mathbf{3}$ Iteration maps of falling disks, constructed as follows. A disk was dropped from rest with some initial angle $\theta_{0}$. From the resulting digitized motion, a sequence of angles at the extrema of motion was measured. Each angle serves as an initial angle $\theta_{\mathrm{i}}$ for the next final angle $\theta_{\mathrm{f}}$. By starting out at several different values of $\theta_{0}$, a rather wide range of angles could be mapped out. To avoid transients, we did not include the initial angle from which the disk was dropped at rest in constructing these maps, making small initial angles sometimes difficult to achieve-the angle after even the first iteration was often already large. Sequence a-d represents a roughly vertical slice through ${ }^{*}$ - Re space, and $\mathbf{e}$-h a horizontal slice. The curves are guides to the eye. The diagonal dashed lines of slope one meet the curves at the fixed points where $\theta_{f}=\theta_{i}$. 3d. A similar route to chaos is observed when taking horizontal slices through the phase diagram, as in $\mathrm{e}-\mathrm{h}$ in Fig. 2, corresponding to maps shown in Fig. 3e-h.

Pomeau and Manneville classify three types of intermittency depending on the way fixed points become unstable ${ }^{15}$. Most experimental systems exhibiting intermittency appear to be of one of these three types. This classification, however, does not cover maps that are not differentiable around fixed points ${ }^{16}$. Here, a fixed point can lose its stability by colliding with a discontinuity in the map. After passing the point of discontinuity, the iteration is reinjected 'stochastically' into the laminar region. We observe this 'type V' intermittency in our experiments.

As we enter the chaotic regime, a discontinuity indeed develops in the map (Fig. 3c, d) at that value of $\theta_{i}=\theta_{c}$ which yields $\theta_{\mathrm{f}}=90^{\circ}$. For $\theta_{\mathrm{i}}>\theta_{\mathrm{c}}$, the disk will tumble several times before being reinjected at some angle less than $\theta_{\mathrm{c}}$. As this tumbling is deterministic, we expect that the map still exists - but is extremely complicated - for angles $\theta_{\mathrm{i}}>\theta_{\mathrm{c}}$. This part of the map is shown schematically in Fig. $3 \mathrm{~d}$ as a rapidly fluctuating line. In this sense the map is essentially discontinuous at $\theta_{c}$.

We now turn to the continuous tumbling regime, observed at very large values of $I^{*}$. This regime cannot be analysed in the same way as the previous three, as the disk never comes to rest and hence no values of $\theta_{\mathrm{i}}$ are defined. It is not clear if this tumbling behaviour is periodic in nature. The analysis of Maxwell (see, for example, ref. 17) indicates that this might be so; however, direct video observation on lead disks shows some departure from periodicity (Fig. 1d). Also, the velocity of a tumbling disk's centre of mass can vary by large ( $\sim 60 \%)$ amounts from the mean value, in an apparently unpredictable manner ${ }^{14}$. These two observations suggest that the rotational period in the tumbling regime is not constant. It is thus possible that the tumbling regime is also characterized by chaotic dynamics, although we have no definitive information on this point. We note that it is possible to access some extremely high values of $I^{*}$ using paper disks in air, which has a very low value of $\rho_{\mathrm{f}}$; to the eye, at least, the rotational period of such disks appears constant. It is therefore possible that at very high values of $I^{*}$ there is another periodic tumbling regime.

Received 12 May; accepted 28 May 1997

1. Viets, H. \& Lee, D. A. Motion of freely falling spheres at moderate Reynolds numbers. Am. Inst. Aeronaut. Astronaut. J. 9, 2038-2042 (1971).

2. Maxwell, J. C. On a particular case of the descent of a heavy body in a resisting medium. Camb. Dublin Math. J. 9, 115-118 (1853).

3. Kajikawa, M. Observation of the falling motion of early snowflakes. J. Meteorol. Soc. Jpn. 60, 797-803 (1982).

4. Allen, J. R. L. Experiments on the settling, overturning and entrainment of bivalve shells and related models. Sedimentology 31, 227-250 (1984).

5. Marchildon, E. K., Clamen, A. \& Gauvin, W. H. Drag and oscillatory motion of freely falling cylindrical particles. Can. J. Chem. Eng. 42, 178-182 (1964).

6. Isaacs, J. L. \& Thodos, G. The free-settling of solid cylindrical particles in the turbulent regime. Can. J. Chem. Eng. 45, 150-155 (1967).

7. Pettyjohn, E. S. \& Christiansen, E. B. Effect of particle shape on free-settling rates of isometric particles. Chem. Eng. Prog. 44, 157-172 (1948).

8. Christiansen, E. B. \& Barker, D. H. The effect of shape and density on the free settling of particles at high Reynolds numbers. Am. Inst. Chem. Eng. J. 11, 145-151 (1965).

9. Aref, H. \& Jones, S. W. Chaotic motion of a solid through ideal fluid. Phys. Fluids A 5, 3026-3028 (1993).

10. Tanabe, Y. \& Kaneko, K. Behaviour of a falling paper. Phys. Rev. Lett. 73, 1372-1375 (1994); Tanabe and Kaneko Reply. 75, 1421 (1995).

11. Mahadevan, L., Aref, H. \& Jones, S. W. Comment on 'Behavior of a Falling Paper'. Phys. Rev. Lett. 75, 1420 (1995).

12. Mahadevan, L. Tumbling of a falling card. C.R. Acad. Sci. Ser. IIb 323, 729-736 (1996).

13. Willmarth, W. W., Hawk, N. E. \& Harvey, R. L. Steady and unsteady motions and wakes of freely falling disks. Phys. Fluids 7, 197-208 (1964); Investigations of the Steady and Unsteady Motion of Freely Falling Disks (Rep. 63-176, USAF Aerospace Res. Lab., Wright-Patterson Air Force Base, Ohio, 1963).

14. Stringham, G. E., Simons, D. B. \& Guy, H. P. The behavior of large particles falling in quiescent liquids. Prof. Pap. US Geol. Surv. 562-C (1969).

15. Schuster, H. G. Deterministic Chaos (VCH, Weinheim, 1995).

16. Bauer, M. et al. New type of intermittency in discontinous maps. Phys. Rev. Lett. 68, 1625-1628 (1992).

17. Lugt, H. J. Autorotation. Annu. Rev. Fluid Mech. 15, 123-147 (1983).

Acknowledgements. We thank D. T. Walker for providing the video equipment. This work was supported in part by the NSF REU Program.

Correspondence should be addressed to S.B.F. (e-mail: field@lamar.colostate.edu). 